\title{
Transitions in Live Video Streaming Services
}

\author{
Björn Richerzhagen \\ TU Darmstadt, Germany \\ richerzhagen@kom.tu- \\ darmstadt.de
}

\author{
Stefan Wilk \\ TU Darmstadt, Germany \\ stefan.wilk@cs.tu- \\ darmstadt.de
}

\author{
Julius Rückert \\ TU Darmstadt, Germany \\ rueckert@ps.tu- \\ darmstadt.de
}

\author{
Denny Stohr \\ TU Darmstadt, Germany \\ denny.stohr@cs.tu- \\ darmstadt.de
}

\author{
Wolfgang Effelsberg \\ TU Darmstadt, Germany \\ wolfgang.effelsberg@cs.tu- \\ darmstadt.de
}

\begin{abstract}
This work shows a demonstration on the work of the Collaborative Research Center 1053 pursuing Multi-Mechanism Adaptation for the Future Internet. Our prototype shows a live video streaming system on mobile devices featuring Peer-to-Peer (P2P) and Client/Server delivery concepts. The focus is to showcase seamless transitions between those mechanisms, depending on the monitored system state. For this, our system streams video from a central server to mobile devices connected by a WiFi Access Point. The system executes transitions between client/server delivery and a P2P streaming overlay based on the number of clients streaming the video. For demonstration purposes, transitions can be triggered either automatically or manually. The transitions between both delivery schemes and the clients' states are monitored and visualized on a central UI.
\end{abstract}

\section{Categories and Subject Descriptors}

C.2.4 [Computer-Communication Networks]: Distributed Systems

\section{Keywords}

P2P; transitions; Client-Server; Monitoring

\section{INTRODUCTION}

Today, a variety of events attract large numbers of live streaming viewers. For some of these live streams access rates can be predicted up-front, others show a more spontaneous but fast increase in the number of parallel viewers. Our research demonstration and the research project addresses those spontaneous and rarely predictable events. In such scenarios, delivering high quality video streams in time cannot be guaranteed - especially when considering mobile networks.

We propose an adaptive streaming system, that showcases a set of mechanisms to mitigate these limitations. The

Permission to make digital or hard copies of part or all of this work for personal or classroom use is granted without fee provided that copies are not made or distributed for profit or commercial advantage, and that copies bear this notice and the full citation on the first page. Copyrights for third-party components of this work must be honored. For all other uses, contact the owner/author(s). Copyright is held by the author/owner(s).

VideoNext December 2, Sydney, Australia

ACM 978-1-4503-3281-1/14/12.

http://dx.doi.org/10.1145/2676652.2683466. system is composed of Peer-to-Peer (P2P) and Client/Server $(\mathrm{C} / \mathrm{S})$ delivery mechanisms that can be switched at runtime. We refer to the replacement of whole networking mechanisms during runtime, such as streaming mechanisms, as transitions. Those transitions enable the streaming system to adapt to a large range of scenarios and conditions such as a sudden increase or decrease in the number of users. Our demonstration illustrates that the replacement of a whole delivery mechanism can be achieved seamlessly without degrading the user experience.

\section{STREAMING OVERLAY}

Modern streaming services have to handle high scalability demands. In most cases, a simple C/S-based system offers the resources required by streaming users but is not scalable to thousands of parallel views. The P2P dissemination paradigm on the other hand offers promising scalability properties, as every client contributes resources to keep the system working. However, P2P systems are expensive in terms of overhead and control based on the number of concurrent accesses. In contrast to existing hybrid P2P-based video dissemination services such as ToMo [1] or mTreebone [2] this demonstration shows a transition-enabled framework for networking mechanisms on the basis of a live video streaming service. Transition-enabled means in this context that $\mathrm{C} / \mathrm{S}$ as well as different P2P-based video dissemination protocols can coexist and are used when environmental conditions favor their selection. The framework is not limited to an exchange of topologies such as it is demonstrated in this work, but allows to switch arbitrary networking mechanisms if they comply to specified interfaces.

This demonstration implements an adaptation mechanism to seamlessly switch between two content dissemination mechanisms: a central C/S and a decentralized P2P-based dissemination scheme. With this it circumvents issues resulting from significantly varying environmental conditions. Streaming should be continued in a consistent quality for all users even though the operations on the network change. The used P2P system is based on Wichtlhuber et al.'s TRANSIT [3]. The $\mathrm{C} / \mathrm{S}$ system follows a classical star topology, where joining nodes contact a server and are provided with small video segments, so called chunks by only this central source. The P2P-based mechanism allows clients to retrieve video chunks from the server but additionally from each available other node. Therefore, a tracking functionality is hosted on the central server to provide node contacts used for the initial 
neighborhoods in the P2P topology, while the exchange of media chunks is then done completely decentralized. As [3] has shown in live streaming scenarios: a tree topology is beneficial to reduce delay between streaming nodes due to the push-based delivery of chunks. Thus, the system aims to arrange clients in a tree, but allows an hybrid video chunk exchange in cases of high churn or if chunks are dropped due to unreliable UDP transmissions. In such cases, the client can request chunks in a pull-based, mesh-like manner from each other node currently in its neighborhood. Transitions and mechanism reconfigurations are shown between $\mathrm{C} / \mathrm{S}$ and $\mathrm{P} 2 \mathrm{P}$, but the P2P-based system allows further replacements of tree and mesh-topologies.

\section{PLANNING AND EXECUTION}

Besides the streaming overlays and mechanisms the demonstration prototype of the research project includes a monitoring, planning, and execution framework allowing sound decisions on when to transition between networking mechanisms. Therefore, the prototype implements a centralized monitoring approach. It allows to gather metrics from each streaming device on the number of nodes involved in streaming one specific live video, the throughput on the up- and downlink, available energy of each client and the streaming quality assessed by the number of stalls. The data is then used by the central video server to visualize the topology and associated metrics. Based on the measured metrics, transition rules can be defined to execute transitions between individual networking mechanisms automatically. In a P2Pbased video streaming system, this involves topology and its management, scheduling, or neighborhood management.

\section{SCENARIO AND DEMONSTRATION}

The demonstration software of the Collaborative Research Centre 1053 illustrates the rising need for efficient, selfadaptive distributed systems, using the case-study of video delivery to mobile devices. It offers a toy model including the central video streaming server represented by a laptop, the wireless network depicted by the WiFi Access Point and five Nexus 7 tablets accessing video from the central server. The central, resource-limited video streaming server offers a continuous video stream. Depending on the interest in the content nodes join the system to watch the stream. The first clients joining the streaming system retrieve the video in a continuously high quality. As more and more nodes are joining the network, the available resources (uplink capacity of the server) are depleted. The system as explained in Section 2 is designed to replace whole mechanisms and streaming approaches without interfering with the application layer - in this case the video playback. As the system is centrally monitored, it can identify a resource bottleneck. When detecting such a bottleneck, it selects an appropriate mechanism for highly scalable video streaming - in this case a P2P overlay - and switches to the new mechanism. During this switch, continuous playback is ensured even though the underlying streaming mechanism is replaced. In one demonstration setting the automatic execution of transitions is disabled initially. Based on a toggle button users can enor disable the automatic execution of transitions. If the transition capabilities are disabled, the user will see reoccurring stalling as the central server's upload capacities are limited. A central visualization as depicted in Figure 1b illustrates

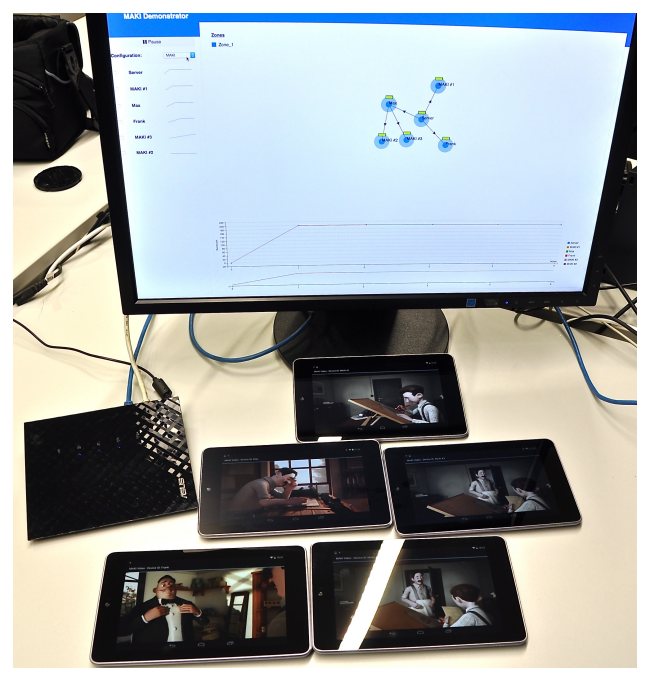

(a) Demonstration setup

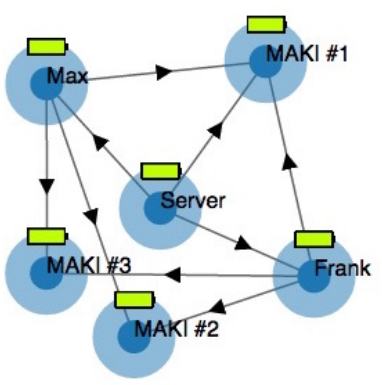

(b) Overview of the streaming topology

metrics of each device as well as aggregated up- and download capacities. Additionally, the current streaming topology is depicted allowing users to easily identify whether the system is currently streaming in $\mathrm{C} / \mathrm{S}$ or $\mathrm{P} 2 \mathrm{P}$ mode. An overview of the setup, consisting of a laptop representing the central streaming server and the Nexus 7 tablets representing the mobile streaming nodes is given in Figure 1a.

\section{Acknowledgement}

This work has been funded by the DFG as part of the CRC 1053 MAKI.

\section{REFERENCES}

[1] S. Awiphan, Z. Su, and J. Katto. ToMo: A Two-Layer Mesh/Tree Structure for Live Streaming in P2P Overlay Network. In 2010 7th IEEE Consumer Communications and Networking Conference, pages 1-5, 2010.

[2] F. Wang, Y. Xiong, and J. Liu. mTreebone: A Hybrid Tree/Mesh Overlay for Application-Layer Live Video Multicast. In IEEE 27th International Conference on Distributed Computing Systems (ICDCS '07), 2007.

[3] M. Wichtlhuber, B. Richerzhagen, J. Rückert, and D. Hausheer. TRANSIT: Supporting transitions in Peer-to-Peer live video streaming. In 2014 IFIP Networking Conference, pages 1-9, 2014. 